\title{
Radical radiotherapy for stage I/II non-small cell lung cancer in patients not sufficiently fit for or declining surgery (medically inoperable): a systematic review
}

\author{
N P Rowell, C J Williams
}

\begin{abstract}
Objectives-To determine the effectiveness of radical radiotherapy in medically inoperable stage I/II non-small cell lung cancer (NSCLC) and the extent of treatment related morbidity.

Methods-Randomised trials were sought by electronically searching the Cochrane Clinical Trials Register, and both randomised and non-randomised trials were sought by searching Medline and Excerpta Medica (Embase). Further studies were identified from references cited in those papers already identified by electronic searching. The studies included were those of patients of any age with stage I/II NSCLC receiving radiotherapy at a dose of $>40 \mathrm{~Gy}$ in 20 fractions over 4 weeks
\end{abstract} or its radiobiological equivalent.

Results-Two randomised and 35 nonrandomised studies were identified. One randomised and nine non-randomised studies did not meet the selection criteria, leaving one randomised and 26 nonrandomised studies for analysis. In the randomised trial 2 year survival was higher following continuous hyperfractionated accelerated radiotherapy (CHART; 37\%) than following $60 \mathrm{~Gy}$ in 30 fractions over 6 weeks $(24 \%)$. An estimated 2003 patients were included in the 26 non-randomised studies; overall survival was $22-72 \%$ at 2 years, $17-55 \%$ at 3 years, and $0-42 \%$ at 5 years. Following treatment, $11-43 \%$ of patients died from causes other than cancer. Cancer specific survival was $54-93 \%$ at 2 years, $22-56 \%$ at 3 years, and $13-39 \%$ at 5 years. Complete response rates were $33-61 \%$ and local failure rates were $6-70 \%$. Distant metastases developed in approximately $25 \%$ of patients. Better response rates and survival were seen in those with smaller tumours and in those receiving higher doses although the reasons for prescribing higher doses were not clearly stated. The outcome was worse in those with prior weight loss or poor performance status. Assessment of treatment related morbidity and effects on quality of life and symptom control were inconclusive because of the lack of prospective evaluation and paucity of data.

Conclusions-No randomised trials compared a policy of immediate radical radiotherapy with palliative radiotherapy given when patients develop symptoms. In the absence of such trials, radical radiotherapy appears to result in a better survival than might be expected had treatment not been given. A substantial, though variable, proportion of patients died during follow up from causes other than cancer. The optimal radiation dose and treatment technique (particularly with respect to mediastinal irradiation) remain uncertain.

(Thorax 2001;56:628-638)

Keywords: radiotherapy; lung cancer; dosage; review

Background

In general, surgery is believed to offer the best prospects for cure for early stage non-small cell lung cancer (NSCLC). In spite of the intention to consider all patients with stage I/II disease for surgery, there are those who, although technically operable, either refuse surgery or are considered inoperable because of insufficient respiratory reserve, cardiovascular disease, or general frailty. This group may therefore be considered "medically inoperable". Some respiratory physicians refer these patients for radical radiotherapy while others believe that radiotherapy has little to offer and adopt a watch policy, referring patients for palliative radiotherapy only when they become symptomatic.

Although there is no evidence from randomised trials to support the use of radical radiotherapy for stage I/II NSCLC, it is the perception of most clinical oncologists (radiotherapists) that patients should receive radical, as opposed to palliative, treatment. ${ }^{1}$

The revised International System for Staging Lung Cancer ${ }^{2}$ is based on outcomes in a series of over 5000 patients. Five year survival in T1N0 (stage IA) and T2N0 (stage IB) pathologically (surgically) staged tumours was $67 \%$ and $57 \%$, respectively, while 5 year survival in clinically staged T1N0 and T2N0 tumours was $61 \%$ and $38 \%$, respectively. For those with involvement of hilar nodes (stage IIA), 5 year survival was $55 \%$ and $39 \%$ for pathologically staged $\mathrm{T} 1 \mathrm{~N} 1$ and $\mathrm{T} 2 \mathrm{~N} 1$ tumours but $34 \%$ and $24 \%$ for those clinically staged. Five year survival for T3N0 (stage IIB) tumours was $38 \%$ when pathologically staged and $22 \%$ when clinically staged. The significance of the two sets of outcomes is that surgical patients are, by definition, pathologically staged while patients receiving radical radiotherapy are almost entirely clinically staged- 
that is, without mediastinoscopy or thoracotomy.

These results contrast with an untreated group of 50 patients with stage I/II disease in which all had died by the end of the third year. ${ }^{3}$ Although the survival following radical radiotherapy is thought to lie somewhere between that of untreated patients and patients treated surgically, outcome is also determined by the presence of other medical conditions, principally chronic obstructive pulmonary disease and coronary artery disease, which rendered the majority of individual patients inoperable.

Although current UK guidelines ${ }^{1}$ recommend radical radiotherapy for this group of patients, factors influencing the decision to refer patients for radiotherapy include uncertainty as to whether radiotherapy can alter the natural history of NSCLC and anxieties about the safety of radiotherapy in the presence of poor lung function. The purpose of this review is to summarise the evidence on treatment effectiveness and morbidity to assist those involved in making treatment decisions. The effectiveness of (external beam) radical radiotherapy for stage I/II medically inoperable NSCLC is determined and, where possible, the extent of morbidity resulting from it. Endobronchial brachytherapy or the additional use of chemotherapy are beyond the scope of this review.

\section{Methods}

CRITERIA FOR CONSIDERING STUDIES IN THIS REVIEW

Criteria for inclusion of studies in this review were established prospectively.

Types of studies

Evidence from randomised controlled trials was sought in the first instance. Where this was not available, results were reported from non-randomised studies, bearing in mind that such evidence is prone to bias.

Types of participants

Patients of any age with T1-2N0-1M0 (now defined as stage I/IIA) or T3NOM0 (now defined as stage IIB) NSCLC, ${ }^{2}$ either considered not sufficiently fit for or declining surgery.

\section{Types of intervention}

External beam radiotherapy given with radical intent (defined as an intended dose of at least 40 Gy delivered in 20 daily fractions or its radiobiological equivalent).

Types of outcome measures

Outcome measures included overall and cancer specific survival, local failure rate, response rate (both complete response and combined complete and partial response), deaths from intercurrent illness, and treatment morbidity (with particular respect to spinal cord and lung toxicity)

Cancer specific survival rates (defined as the survival rate calculated after exclusion of those patients known to have died from causes other than cancer) are as quoted in individual studies. Local failure was defined as persistent or recurrent disease at the primary site. Regional failure was defined as persistent or recurrent disease at the hilum or within the mediastinum or supraclavicular fossae. Locoregional failure was defined as failure in either local or regional sites. Complete and partial response rates were defined according to standard UICC criteria. $^{4}$

\section{SEARCH STRATEGY}

Randomised trials were sought by electronically searching the Cochrane Clinical Trials Register and both randomised and nonrandomised trials were sought by searching Medline and Excerpta Medica (Embase). Further studies were identified from references cited in those papers already identified by electronic searching. Searches were updated during July 2000.

\section{METHODS OF THE REVIEW}

Each study identified by the search strategy was categorised as either randomised or nonrandomised and assessed independently by both reviewers to determine whether it met the inclusion criteria. Any disagreements were resolved by discussion, involving a third party where necessary.

\section{DESCRIPTION OF THE STUDIES}

Two randomised trials were identified, one comparing surgery with radiotherapy ${ }^{5}$ and the other comparing two radiotherapy fractionation schedules (continuous hyperfractionated accelerated radiotherapy (CHART) versus conventionally fractionated radiotherapy). ${ }^{6}$ The surgery versus radiotherapy trial was excluded as a proportion of patients were thought to have had involvement of mediastinal nodes. The CHART trial only met the inclusion criteria with subsequent publication of a subgroup analysis giving survival data for patients with stage I/IIA disease. ${ }^{\text {? }}$

Forty two references to non-randomised studies were identified. ${ }^{8-49}$ Several studies reported the results of treatment with a wide range of radiation doses. In some cases it was not clear whether doses lower than $40 \mathrm{~Gy}$ were used intentionally or were the result of treatment being discontinued before the prescribed dose had been delivered. Studies in which the majority of the patients had received doses greater than 40 Gy were included. Some studies included patients with stage III disease; these were included if sufficient data relevant only to patients with stage I/II were reported. Some studies included patients without a pathological confirmation of the diagnosis; while confirmation of the diagnosis is generally considered to be preferable, it is recognised that a proportion of patients have insufficient respiratory reserve to tolerate a pneumothorax following a CT guided lung biopsy. Studies were considered eligible if the proportion without pathological confirmation did not exceed $10 \%$.

Thirty two references to 26 non-randomised studies $^{8-39}$ form the basis of this review (table 1). Nine studies (10 references) were excluded. The reasons for exclusion were: stage not stated, ${ }^{40}$ radiation dose less than $40 \mathrm{~Gy},{ }^{42}{ }^{47}$ 
Table 1 Pretreatment characteristics of patients

\begin{tabular}{|c|c|c|c|c|c|c|}
\hline Reference & $n$ & $\begin{array}{l}\text { Mean/median age } \\
\text { (range) }\end{array}$ & $\begin{array}{l}\text { Mediastinoscopy } \\
(\%)\end{array}$ & $\begin{array}{l}\text { Staged with } C T \\
(\%)\end{array}$ & $\begin{array}{l}\text { Declined surgery } \\
(n)\end{array}$ & $\begin{array}{l}\text { Unfit for surgery } \\
(n)\end{array}$ \\
\hline \multicolumn{7}{|l|}{ Stage I (T1-2NO) } \\
\hline Haffty $(1988)^{8}$ & 43 & $64(51-83)$ & 4 & 9 & 6 & 37 \\
\hline Noordijk $(1988)^{9} \mathrm{a}$ & 50 & & & & & \\
\hline Sandler $(1990)^{10}$ & 77 & $72(44-80+)$ & & 16 & 13 & 64 \\
\hline Ono $(1991)^{11}$ & 38 & Not stated & & 24 & 4 & 34 \\
\hline Kaskowitz $(1993)^{12}$ & 53 & $73(44-86)$ & 8 & All & 10 & 43 \\
\hline Slotman $(1994)^{13 \mathrm{~b}}$ & 47 & $75(57-93)$ & & 55 & 3 & 44 \\
\hline Gauden $(1995)^{14}$ & 347 & $70(34-90)$ & & 87 & Not stated & Not stated \\
\hline Park $(1995)^{15}$ & 15 & 66 & & Not stated & 2 & 13 \\
\hline Krol $(1996)^{16}$ & 108 & $74(56-88)$ & & 86 & 6 & 102 \\
\hline Slotman $(1996)^{17 b}$ & 31 & $75(54-85)$ & & All & 2 & 29 \\
\hline Morita $(1997)^{18 \mathrm{~b}}$ & 149 & $74(50-89)$ & & All & 26 & 123 \\
\hline Jeremic $(1997)^{19}$ & 49 & $63(51-70)$ & & All & 20 & 29 \\
\hline Nguyen-Tan $(1998)^{20 \mathrm{c}}$ & 41 & $69(53-81)$ & 36 & All & 4 & 37 \\
\hline Sibley $(1998)^{21}$ & 141 & $70(46-95)$ & 1 & 90 & 2 & 139 \\
\hline Hayakawa $(1999)^{22 ~ b}$ & 36 & $>70$ & & 67 & 4 & 32 \\
\hline \multicolumn{7}{|l|}{ Stage IIA (T1-2N1) } \\
\hline Salloum $(1990)^{23}$ & 61 & $69^{d}$ & & Some & Not stated & Not stated \\
\hline Rosenthal $(1992)^{24}$ & 62 & $68(46-82)$ & & Some & Not stated & Not stated \\
\hline \multicolumn{7}{|l|}{ Stage I E IIA (T1-2N0-1) } \\
\hline Zhang $(1989)^{25}$ & 42 & $57(37-70)^{\mathrm{d}}$ & & 0 & 12 & 32 \\
\hline Hayakawa $(1992)^{26 \text { a }}$ & 64 & & & & & \\
\hline Schildge $(1993)^{27}$ & 67 & 66 (not stated) & All earlier patients & Later patients only & Not stated & Not stated \\
\hline Graham $(1995)^{28}$ & 103 & $67(43-89)$ & 3 & 76 & Not stated & Not stated \\
\hline Koukourakis $(1995)^{29}$ & 64 & $67^{\mathrm{d}}$ (not stated) & & All & Not stated & Not stated \\
\hline Furuta $(1996)^{30 \mathrm{~b}}$ & 32 & $79(75-86)$ & & Most & 2 & 30 \\
\hline Hayakawa $(1996)^{31}$ & 84 & $>70$ & & Some & 5 & 79 \\
\hline Hayakawa $(1996)^{32 \mathrm{e}}$ & 84 & & & & & \\
\hline King $(1996)^{33}$ a & 11 & & & & & \\
\hline Saunders (1999), ${ }^{6}$ Bentzen $(2000)^{7}$ & 169 & Not stated & & All & Not stated & Not stated \\
\hline Anderson $(2000)^{34}$ & 36 & $68(46-82)$ & & All & Not stated & Not stated \\
\hline \multicolumn{7}{|l|}{ Stage I E IIB (T1-3NO) } \\
\hline Talton $(1990)^{35}$ & 77 & 65 (not stated) & Some & 20 & Not stated & Not stated \\
\hline Dosoretz $(1992),{ }^{36}(1993)^{37 \text { a }}$ & 152 & $74(51-88)$ & & Most & Not stated & Not stated \\
\hline Dosoretz $(1996)^{38}$ & 245 & Not stated & & & 27 & 218 \\
\hline Kupelian $(1996)^{39}$ & 59 & Not stated & & All & Not stated & Not stated \\
\hline
\end{tabular}

Data from individual studies have not been included in the table when these data have been included in a subsequent or duplicate series. Data from randomised trial shown in bold.

${ }^{a}$ Updated series: patients from Noordijk $(1988)^{9}$ were included in Krol (1996), ${ }^{16}$ from Hayakawa (1992) ${ }^{26}$ in Hayakawa (1996), ${ }^{31}$ from Dosoretz $(1992)^{36}$ and (1993) ${ }^{37}$ in Dosoretz (1996), ${ }^{38}$ and from King $(1996)^{33}$ in Sibley (1998). ${ }^{21}$

${ }^{b}$ Overlapping series: some patients reported by Hayakawa $(1996)^{31}$ and Furuta $(1996)^{30}$ were included in a series with stage I tumours reported by Morita (1997) ${ }^{18}$ and in a series of peripheral stage I tumours reported by Hayakawa (1999). ${ }^{22}$ Some patients reported in Hayakawa (1996) ${ }^{31}$ were included in a subset of elderly patients with stage I/IIA tumours reported by Furuta (1996). ${ }^{30}$ Slotman $(1984)^{13}$ and $(1987)^{17}$ reported patients from overlapping time periods.

'Published data supplemented by data from authors (Nguyen-Tan, personal communication).

${ }^{\mathrm{d} A g e}$ ranges are those of a larger group which included a proportion of patients with stage III tumours.

${ }^{e}$ Duplicate series: Hayakawa $(1996)^{32}$ reported the same results as Hayakawa (1996). ${ }^{31}$

inclusion of patients with small cell lung cancer, 404344 more than $10 \%$ of patients without cytological or histological confirmation, ${ }^{43}{ }^{44}$ studies of patients with stages I-III but insufficient outcome data relating solely to those with stage I/II. ${ }^{44-49}$

\section{METHODOLOGICAL QUALITY OF INCLUDED}

STUDIES

The randomised (CHART) trial $^{6}$ was a multicentre study with randomisation performed by the method of minimisation by telephone call to a central trials office; data analysis was performed on an intention-to-treat basis and exclusions were solely on the basis of ineligibilitythat is, failing to meet the entry criteria with regard to pathological diagnosis or performance status. The 26 non-randomised studies were all retrospective reviews (table 1)..$^{8-39}$

\section{Duplicate reporting}

The extent of duplicate reporting was uncertain as some authors did not cite previous reports which included patients from the same centre over the same or an overlapping time period. As far as possible, these are identified in table 1 .
Patient characteristics

Most of the non-randomised studies recorded details of age, stage, the use of CT imaging and mediastinoscopy for staging, and numbers of patients declining or considered insufficiently fit for surgery (table 1). Only those patients with WHO performance status $0-1$ were eligible for the CHART trial. ${ }^{6}$ Data on performance status and weight loss were included in 16 and five non-randomised studies, respectively (table 2).

\section{End points}

A range of end points was used and in several studies the end points sought by this review were not reported. Different definitions of local and locoregional failure were used or were not defined. For example, it was not always clear whether in-field recurrences were local or regional or whether intrathoracic recurrences were in fact local, regional, or distant.

\section{Adverse event reporting}

The CHART trial ${ }^{6}$ recorded treatment morbidity prospectively using 4- or 5-point scales. While almost all the non-randomised studies appeared to report major morbidity, the reporting of treatment morbidity was, in general, poor. There was grading of morbidity 
Table 2 Patient characteristics

\begin{tabular}{lll}
\hline Reference & Performance status & Weight loss \\
\hline Saunders (1999), ${ }^{6}$ Bentzen (2000) & All 0-1 & - \\
Sandler $(1990)^{10}$ & $63 \% 0-1$ & - \\
Kaskowitz $(1993)^{12}$ & $91 \% 70-100$ & $>5 \%$ in $32 \%$ \\
Slotman $(1994)^{13}$ & $64 \% 70-80 ; 32 \% 90-100$ & - \\
Gauden $(1995)^{14}$ & $74 \% 0-1$ & - \\
Park $(1995)^{15}$ & $13 \% 0-1$ & - \\
Krol $(1996)^{16}$ & All 70-100 & - \\
Slotman $(1996)^{17}$ & $65 \% 70-80 ; 23 \% 90-100$ & $>5 \%$ in $16 \%$ \\
Jeremic $(1997)^{19}$ & $27 \% 70-80 ; 73 \% 90-100$ & Present in $26 \%$ \\
Sibley $(1998)^{21}$ & - & - \\
Hayakawa $(1999)^{22}$ & $97 \% 0-1$ & $>5 \%$ in $40 \%$ \\
Rosenthal $(1992)^{24}$ & $66 \% 0-1$ & Present in $33 \%$ \\
Graham $(1995)^{28}$ & $87 \% 0-1$ & - \\
Koukourakis $(1995)^{29}$ & All 70-100 & - \\
Furuta $(1996)^{30}$ & $81 \% 0-1$ & - \\
Hayakawa $(1996)^{31}$ & $63 \% 0-1$ & - \\
Anderson $(2000)^{34}$ & $56 \% 0-1$ & - \\
Kupelian $(1996)^{39}$ & $57 \% 70-100$ & \\
\hline
\end{tabular}

Performance status is graded by either the ECOG/WHO or Karnofsky scales; good performance status patients are those with ECOG/WHO 0-1 or Karnofsky scores of 70-100. Randomised tria shown in bold.

Table 3 Radiotherapy details

\begin{tabular}{|c|c|c|}
\hline Reference & Radiation dose ( $G y /$ no of fractions $(F)$ ) & $\begin{array}{l}\text { Mediastinum } \\
\text { included } \\
\text { (\% patients) }\end{array}$ \\
\hline Haffty $(1988)^{8}$ & $\begin{array}{l}\text { Continuous: median } 59 \text { Gy in } 2 \text { Gy fractions or } \\
\text { Split: median } 54 \text { Gy in median } 2.75 \text { Gy fractions }\end{array}$ & $88 \%$ \\
\hline Sandler $(1990)^{10}$ & Median $60 \mathrm{~Gy} / 30 \mathrm{~F} / 6$ weeks & $90 \%$ \\
\hline Ono $(1991)^{11}$ & $60-70 \mathrm{~Gy} / 30-35 \mathrm{~F}$ split over $8-10$ weeks & $32 \%$ \\
\hline Kaskowitz $(1993)^{12}$ & 40-79 Gy (median 63 Gy) & $89 \%$ \\
\hline Slotman $(1994)^{13}$ & $32 \mathrm{~Gy} / 6 \mathrm{~F}$ twice weekly, $40 \mathrm{~Gy} / 10 \mathrm{~F}$ split, 48 & \\
\hline Gauden $(1995)^{14}$ & $50 \mathrm{~Gy} / 20 \mathrm{~F} / 4$ weeks & $100 \%$ \\
\hline Park $(1995)^{15}$ & $60 \mathrm{~Gy} / 30 \mathrm{~F}$ & Not stated \\
\hline Krol $(1996)^{16}$ & $60 \mathrm{~Gy} / 20 \mathrm{~F}$ split over 7 weeks or $65 \mathrm{~Gy} / 26 \mathrm{~F}$ daily & $0 \%$ \\
\hline Slotman $(1996)^{17}$ & $48 \mathrm{~Gy} / 12 \mathrm{~F}$ daily & $0 \%$ \\
\hline Morita $(1997)^{18}$ & $55-74$ Gy mostly in 2 Gy fractions & $44 \%$ \\
\hline Jeremic $(1997)^{19}$ & $69.6 \mathrm{~Gy} / 58 \mathrm{~F}$ treatment twice daily & Hilum included \\
\hline Nguyen-Tan $(1998)^{20}$ & $40 \mathrm{~Gy} / 10 \mathrm{~F}$ split-64 Gy/32F & Not stated \\
\hline Sibley $(1998)^{21}$ & $\begin{array}{l}50-80 \text { Gy in } 1.2-3 \text { Gy fractions; } 15 \text { patients } \\
\text { treated twice daily }\end{array}$ & $73 \%$ \\
\hline Hayakawa $(1999)^{22}$ & $48-81$ Gy in 2 Gy daily fractions & $28 \%$ \\
\hline Salloum $(1990)^{23}$ & $15-66$ Gy $(71 \% \geqslant 50$ Gy; $25 \%$ split course $)$ & Not stated \\
\hline Rosenthal $(1992)^{24}$ & $18-65$ Gy (median 60 Gy) & $100 \%$ \\
\hline Zhang $(1989)^{25}$ & $55-70 \mathrm{~Gy}$ in $6-7$ weeks & $100 \%$ \\
\hline Schildge $(1993)^{27}$ & $60 \mathrm{~Gy} / 30 \mathrm{~F}$ & Not stated \\
\hline Graham $(1995)^{28}$ & Median 60 Gy/30F daily (range $18-60$ Gy) & $80 \%$ \\
\hline Koukourakis $(1995)^{29}$ & $\begin{array}{l}\text { NTD } 48-64 \text { Gy in } 2-4 \text { Gy fractions over 3-10 } \\
\text { weeks }\end{array}$ & $100 \%$ \\
\hline Furuta $(1996)^{30}$ & $60-80$ Gy/6-8 weeks & $81 \%$ \\
\hline Hayakawa $(1996)^{31}$ & $\begin{array}{l}60-74 \text { Gy in } 2 \text { Gy fractions; } 10 \text { patients received } \\
80-90 \text { Gy }\end{array}$ & $85 \%$ \\
\hline $\begin{array}{l}\text { Saunders (1999), }{ }^{6} \\
\text { Bentzen }(2000)^{7}\end{array}$ & $\begin{array}{l}60 \text { Gy/30F daily or } 54 \text { Gy/36F/12 days } \\
\text { (CHART) }\end{array}$ & $100 \%$ \\
\hline Anderson $(2000)^{34}$ & $45-55 \mathrm{~Gy} / 15-16 \mathrm{~F}$ daily & Not stated \\
\hline Talton $(1990)^{35}$ & $60 \mathrm{~Gy} / 30 \mathrm{~F} / 6$ weeks & $100 \%$ \\
\hline $\begin{array}{l}\text { Dosoretz }(1992),{ }^{36}(1993),{ }^{37} \\
(1996)^{38}\end{array}$ & $45-75$ Gy in $1.8-2$ Gy fractions & Most \\
\hline Kupelian (1996) ${ }^{39}$ & Median 63 Gy & $72 \%$ \\
\hline
\end{tabular}

NTD = nominal total dose.

Randomised trial shown in bold. relating to the 169 patients with stage I/IIA disease showed a similar degree of benefit with 2 year survival improving from $24 \%$ for conventional radiotherapy to $37 \%$ with CHART. Four year survival improved from $12 \%$ to $18 \%$. Most of the patients $(130 / 169$, $77 \%)$ had T2N0 tumours; 33 (20\%) were T1N0 and only six (4\%) were stage IIA. A two phase technique was used with elective mediastinal irradiation in the first phase.

\section{NON-RANDOMISED STUDIES}

Patient characteristics

Allowing for duplicate reporting, an estimated 2003 patients were included in 26 nonrandomised studies (table 1). ${ }^{8-39}$ Individual studies were of varying size, ranging from 15 to 347 patients (median study size 60 patients). In two studies there were nine of $108^{16}$ and four of $103^{28}$ without pathological confirmation of the diagnosis.

Age: the patients had a wide age range (34-95 years; table 1). Some studies reported median age while others reported the mean age. Taken together, median/mean ages in individual studies ranged from 57 to 79 years (median 70).

Staging: in the series reported before 1992 less than a quarter of patients had had CT imaging whereas, in those reported since, CT imaging had been performed in most (table 1). Mediastinoscopy was mentioned as being used in seven studies.

Location of stage I tumours: two studies included only peripheral tumours ${ }^{16}{ }^{22}$ and, in a further study, $87 \%$ were located peripherally. ${ }^{17}$

Performance status and weight loss: most of the patients were of good performance status (ECOG/WHO 0-1, range $13-97 \%$ but $>50 \%$ in all but one study; Karnofsky 70-100, range 57-100\%; table 2). Ten studies did not report performance status. The presence of weight loss was noted in only five studies ( $>5 \%$ of body mass in three studies but undefined in two) and was reported by $16-40 \%$ of patients (table 2 ).

Proportion declining surgery $v$ those considered not sufficiently fit: this was reported in 17 studies $(n=1233$ patients, table 1$)$ in which 148 (12\%) had declined surgery. Reasons for inoperability were given for 1003 patients and included pulmonary disease in $45 \%$, cardiac disease in $13 \%$, other unspecified medical problems in $20 \%$, poor general condition in $9 \%$, age in $11 \%$, and other cancer in $1 \%$.

Comorbidity: this was recorded separately from reasons for inoperability in two studies, ${ }^{13}{ }^{28}$ in one of which ${ }^{28}$ only 13 of 103 patients had no comorbidity. A better outcome was reported in those with normal cardiorespiratory function. ${ }^{27}$

Treatment details: details of radiotherapy fractionation and whether the mediastinum was included in the treated volume are shown in table 3.

\section{Survival}

Overall survival: overall survival in the studies was $50-93 \%$ at 1 year, $22-72 \%$ at 2 years, $17-55 \%$ at 3 years, and $0-42 \%$ at 5 years. Median survival varied from 15 to 33 months 
Table 4 Overall and cancer specific survival

\begin{tabular}{|c|c|c|c|c|c|c|c|c|c|c|c|c|c|c|}
\hline \multirow[b]{2}{*}{ Reference } & \multirow{2}{*}{$\begin{array}{l}\text { Intercurrent } \\
\text { deaths (\%) }\end{array}$} & \multirow[b]{2}{*}{$n$} & \multirow{2}{*}{$\begin{array}{l}\text { Clinical } \\
\text { subset }\end{array}$} & \multirow{2}{*}{$\begin{array}{l}\text { Median } \\
\text { survival } \\
\text { (months) }\end{array}$} & \multicolumn{4}{|c|}{ Overall survival (\%) } & \multicolumn{4}{|c|}{ Cancer specific survival (\%) } & $\begin{array}{l}\text { Disease } \\
\text { surviva }\end{array}$ & $\begin{array}{l}\text { ree } \\
(\%)\end{array}$ \\
\hline & & & & & 1 year & 2 year & 3 year & 5 year & 1 year & 2 year & 3 year & 5 year & 2 year & 5 year \\
\hline Stage I (T1-2NO) & & & & & & & & & & & & & & \\
\hline Haffty $(1998)^{8}$ & NS & 43 & & 28 & & & 36 & 21 & & & & & & \\
\hline & & 11 & Continuous & 52 & & & & 45 & & & & & & \\
\hline & & 32 & Split & 27 & & & & 12 & & & & & & \\
\hline Sandler $(1990)^{10}$ & 13 & 77 & & 20 & & & 17 & & & & 22 & & & \\
\hline & & 25 & $<3 \mathrm{~cm}$ & & & & 25 & & & & 30 & & & \\
\hline & & 41 & $3-6 \mathrm{~cm}$ & & & & 13 & & & & 17 & & & \\
\hline & & 9 & $>6 \mathrm{~cm}$ & & & & 0 & & & & 0 & & & \\
\hline Ono $(1991)^{11}$ & NS & 38 & & NS & 84 & 68 & 55 & 42 & & & & & & \\
\hline Kaskowitz $(1993)^{12}$ & 25 & 53 & & 21 & & 43 & 19 & 6 & & 54 & 33 & 13 & & \\
\hline Slotman $(1994)^{13}$ & 43 & 47 & & 20 & 70 & & 33 & 15 & 90 & & 53 & 32 & & \\
\hline Gauden $(1995)^{14}$ & 12 & 347 & & 28 & & 54 & 40 & 27 & & & & & 41 & 23 \\
\hline & & 167 & $T 1$ & & & & & 32 & & & & & & \\
\hline & & 180 & $T 2$ & & & & & 21 & & & & & & \\
\hline Park $(1995)^{15}$ & NS & 15 & & 15 & & 22 & & 0 & & & & & & \\
\hline Krol $(1996)^{16}$ & 30 & 108 & & 23 & 75 & 49 & 31 & 15 & 81 & 58 & 42 & 31 & & \\
\hline & & 89 & $\leqslant 4 \mathrm{~cm}$ & & & & 35 & & & & 40 & & & \\
\hline & & 19 & $>4 \mathrm{~cm}$ & & & & 11 & & & & 13 & & & \\
\hline Slotman $(1996)^{17}$ & 42 & 31 & & 33 & 81 & 72 & 42 & 8 & & 93 & $76^{\mathrm{a}}$ & & & \\
\hline Morita $(1997)^{18}$ & 13 & 149 & & 27 & 78 & & 34 & 22 & & & & & & \\
\hline & & & $<4 \mathrm{~cm}$ & & & & & 25 & & & & & & \\
\hline & & & $>4 \mathrm{~cm}$ & & & & & 17 & & & & & & \\
\hline Jeremic $(1997)^{19}$ & 12 & 49 & & 33 & & & & 30 & & & & & & 41 \\
\hline & & 25 & $T 1$ & 40 & & 76 & & 37 & & & & & & \\
\hline & & 24 & $T 2$ & 24 & & 50 & & 24 & & & & & & \\
\hline Nguyen-Tan $(1998)^{20}$ & 20 & 41 & & 23 & & 41 & & & & & & & & \\
\hline & & 19 & $T 1$ & 26 & & 63 & & & & & & & & \\
\hline & & 22 & $T 2$ & 14 & & 24 & & & & & & & & \\
\hline Sibley $(1998)^{21}$ & 33 & 141 & & 18 & & 39 & & 13 & & 60 & & 32 & & \\
\hline Hayakawa $(1999)^{22}$ & NS & 36 & & NS & & & 42 & 23 & & & 56 & 39 & & \\
\hline Stage IIA (T1-2N1) & & & & & & & & & & & & & & \\
\hline Salloum $(1990)^{23}$ & NS & 61 & & 18 & & & & 22 & & & & & & \\
\hline Rosenthal $(1992)^{24}$ & NS & 62 & & 18 & 70 & 33 & 20 & 12 & & & & & & \\
\hline & & & $P S 0-1$ & 23 & 83 & 49 & 28 & & & & & & & \\
\hline & & & PS 2-3 & 6 & & & & & & & & & & \\
\hline Stage I \& IIA (T1-2N0-1) & & & & & & & & & & & & & & \\
\hline Zhang $(1989)^{25}$ & 14 & 42 & & NS & 93 & & 52 & 31 & & & & & & \\
\hline Schildge $(1993)^{27}$ & NS & 67 & & 17 & 55 & & 19 & 5 & & & & & & \\
\hline & & 35 & $N C R F$ & & 69 & & 21 & 11 & & & & & & \\
\hline Graham $(1995)^{28}$ & 25 & 103 & & 16 & & 35 & & 13 & & & & & & \\
\hline & & 35 & $T 1^{b}$ & & & & & 29 & & & & & & \\
\hline & & 68 & $T 2^{b}$ & & & & & 4 & & & & & & \\
\hline Koukourakis $(1995)^{29}$ & NS & 64 & NTD & NS & & & & & & & & & & \\
\hline & & & $56-64 G y$ & & & & & & & & & & & 30 \\
\hline & & & $48-55 G y$ & & & & & & & & & & & 12 \\
\hline Furuta $(1996)^{30}$ & 34 & 32 & & NS & & 40 & & 16 & & 57 & & 36 & 43 & 23 \\
\hline Hayakawa $(1996)^{31}$ & NS & 84 & & NS & & & & & & & & & & \\
\hline & & 28 & T1-2NO & & & 74 & & 31 & & & & & & \\
\hline & & 56 & $T 1-2 N 1$ & & & 40 & & 19 & & & & & & \\
\hline & & 48 & $<5 \mathrm{~cm}$ & & & & & 29 & & & & & & \\
\hline & & 36 & $\geqslant 5 \mathrm{~cm}$ & & & & & 14 & & & & & & \\
\hline Saunders $(1999),{ }^{6}$ & NS & 169 & $60 \mathrm{~Gy}$ & NS & & 24 & $12^{\mathrm{a}}$ & & & & & & & \\
\hline Bentzen (2000) ${ }^{7}$ & & & CHART & & & 37 & $18^{\mathrm{a}}$ & & & & & & & \\
\hline Anderson $(2000)^{34}$ & NS & 36 & & 12 & 50 & 22 & & 6 & & & & & & \\
\hline Stage I E IIB (T1-3NO) & & & & & & & & & & & & & & \\
\hline Talton $(1990)^{35}$ & NS & 77 & & 18 & 56 & 37 & 21 & 17 & & & & & & \\
\hline Dosoretz $(1992){ }^{36}(1993)^{37}$ & 11 & 152 & & & & 40 & & & & & & & & 9 \\
\hline & & 44 & $T 1$ & $N R$ & & & & & & & & & 55 & \\
\hline & & 63 & $T 2$ & 12 & & & & & & & & & 20 & \\
\hline & & 41 & T3 & 12 & & & & & & & & & 25 & \\
\hline Dosoretz $(1993)^{37}$ & & & $T 160-65 G y$ & & & & & & & & & & $29^{\mathrm{c}}$ & \\
\hline & & & $T 1>65 G y$ & & & & & & & & & & $90^{\mathrm{c}}$ & \\
\hline Dosoretz $(1996)^{38}$ & & 245 & & 16 & & & & 7 & & & & & 28 & 9 \\
\hline & & & $<3 \mathrm{~cm}$ & & & & & & & & & & 38 & \\
\hline & & & $>5 \mathrm{~cm}$ & & & & & & & & & & 23 & \\
\hline Kupelian $(1996)^{39}$ & NS & 19 & $T 1$ & NS & & & & & & & 49 & & & \\
\hline & & 26 & $T 2$ & & & & & & & & 47 & & & \\
\hline & & 14 & $T 3$ & & & & & & & & 26 & & & \\
\hline
\end{tabular}

Intercurrent deaths are deaths known not to be due to cancer (where reported), shown as a percentage of the number of patients undergoing treatment. $\mathrm{NS}=$ not stated; $\mathrm{NR}=$ not reached; $\mathrm{NCRF}=$ normal cardiorespiratory function.

${ }^{a} 4$ year survival; ${ }^{b}$ only $10 \%$ of each subgroup had N1 disease; ${ }^{\mathrm{c}} 3$ year disease free survival.

Randomised trial shown in bold; results of subgroup analyses shown in italics.

(but was not stated in seven studies; table 4 without subgroup analysis).

Survival was better for $\mathrm{T} 1$ than for $\mathrm{T} 2$ tumours (range $29-37 \%$ v $4-24 \%$ at 5 years) ${ }^{12} 1419202836$ and fell progressively as tumour size increased, although different studies used different cut off sizes. ${ }^{1016183138}$ For those with involved hilar nodes, 5 year survival ranged from $12 \%$ to $22 \% .^{2324} 31$ Survival was unaffected by whether or not patients had had CT imaging performed as part of staging. ${ }^{1024}$

Survival appeared to be better in subgroups which received a higher dose in some studies ${ }^{12} 2829{ }^{37}$ but, in another, ${ }^{18}$ the complete response rate was improved with higher doses but survival appeared to be unaffected. In 
contrast, the development of bronchial stenosis with doses higher than 80 Gy may have contributed to better survival with doses in the range $60-69 \mathrm{~Gy}^{31}$

In some studies there was a trend in favour of better overall survival in those aged less than 70 years ${ }^{12}{ }^{14}$ or less than 80 years, ${ }^{18}$ but not in others. ${ }^{10131617242831}$ The presence of comorbidity $^{1328}$ or weight $\operatorname{loss}^{1928}$ affected survival adversely. There were striking differences in outcome according to performance status. Median survival times in the studies with data on performance status was 23-24 months for those with ECOG performance status $0-1$ or Karnofsky scores of 90-100 compared with $6-13$ months for those with ECOG $\geqslant 2^{24} 31$ or Karnofsky scores of $70-80 .{ }^{19}$ In contrast, in one study there was no difference in survival in those with Karnofsky scores $\geqslant 70$ compared with $<70,{ }^{12}$ and in another survival was unaffected by the presence of weight loss. ${ }^{24}$

Differences in survival by histological subtype (squamous, adenocarcinoma, or large cell carcinoma) were noted in only one study in which those with squamous carcinomas had a better outcome. ${ }^{21}$

Disease free survival: the disease free survival was $28-43 \%$ at 2 years and $9-41 \%$ at 5 years (table 4). ${ }^{14} 193038 \mathrm{~A}$ further study attempted to equate dose schedules that incorporated a range of total doses and fraction sizes or the use of split courses by quoting the nominal total dose (NTD) rather than the prescribed dose, and demonstrated better disease free survival in those receiving a higher NTD. ${ }^{29}$

Intercurrent deaths: intercurrent deaths, defined as the proportion of patients undergoing treatment who died from non-cancer causes during the follow up period, are shown in table 4 . There was a wide range of intercurrent death rates $(11-43 \%$, median $25 \%$ ). This is likely to be an underestimate as only those deaths known not to be due to cancer would have been reported.

Cancer specific survival: because of the varying proportions of intercurrent deaths, cancer specific survival may give a more reliable estimate of the effectiveness of treatment. Cancer specific survival in studies reporting data was $54-93 \%$ at 2 years, $22-56 \%$ at 3 years, and $13-39 \%$ at 5 years (table 4 ).

Local control: complete tumour response rates overall were $33-61 \%$ with complete and partial response rates of 80-100\%. ${ }^{11} 1516181924$ Complete response rates declined with increasing tumour size, being $48-52 \%$ in tumours less than $4 \mathrm{~cm}$ in diameter and $20-21 \%$ for those over $4 \mathrm{~cm} .{ }^{16}{ }^{18} \mathrm{~A}$ complete response rate of $60 \%$ was observed in patients receiving 72 Gy compared with $32 \%$ after $60 \mathrm{~Gy}$, although survival was unchanged. ${ }^{18}$ Local failure ranged from $6 \%$ to $70 \%$ and was related to the dose given ${ }^{12} 2125$ and to tumour stage ${ }^{19203639}$ or size $^{31}$ (table 5). In-field, local, or locoregional progression free survival at 3 years was $44-51 \% .^{10}{ }^{12}$

Table 5 Patterns of failure

\begin{tabular}{|c|c|c|c|c|c|c|c|}
\hline \multirow[b]{2}{*}{ Reference } & \multirow[b]{2}{*}{ Subset } & \multirow{2}{*}{$\begin{array}{l}\begin{array}{l}\text { Local failure } \\
(\%)\end{array} \\
\text { Any or first }\end{array}$} & \multicolumn{2}{|c|}{$\begin{array}{l}\text { Regional failure } \\
(\%)\end{array}$} & \multicolumn{2}{|c|}{$\begin{array}{l}\text { Locoregional failure } \\
(\%)\end{array}$} & \multirow{2}{*}{$\begin{array}{l}\begin{array}{l}\text { Distant metastases } \\
(\%)\end{array} \\
\text { Any or first }{ }^{d}\end{array}$} \\
\hline & & & Isolated & Any & $\begin{array}{l}\text { Isolated or } \\
\text { first } t^{d}\end{array}$ & Any & \\
\hline \multicolumn{8}{|l|}{ Stage I (T1-2N0) } \\
\hline Haffty $(1988)^{8}$ & & & & & & 39 & \\
\hline Sandler $(1990)^{10}$ & & & & & & $43^{\mathrm{a}}$ & \\
\hline \multirow{3}{*}{ Kaskowitz $(1993)^{12}$} & & 63 & & & & & 40 \\
\hline & $<65 G y$ & 50 & & & & & \\
\hline & $>65 G y$ & 25 & & & & & \\
\hline Slotman $(1994)^{13}$ & & & & & $26^{\mathrm{b}}$ & & 21 \\
\hline $\operatorname{Krol}(1996)^{16}$ & & 68 & 2 & 15 & & & 33 \\
\hline Slotman $(1996)^{17}$ & & 6 & 3 & 6 & & & 16 \\
\hline Morita $(1997)^{18}$ & & 44 & & & & & \\
\hline \multirow[t]{3}{*}{ Jeremic $(1997)^{19}$} & & 45 & 0 & 11 & & & 25 \\
\hline & $T 1$ & 29 & & & & & \\
\hline & $T 2$ & 62 & & & & & \\
\hline \multirow[t]{3}{*}{ Nguyen-Tan $(1998)^{20}$} & & 41 & & & & & 22 \\
\hline & $T 1$ & 32 & & & & & \\
\hline & $T 2$ & 50 & & & & & \\
\hline Sibley $(1998)^{21}$ & & 22 & 3 & 7 & & & 23 \\
\hline Hayakawa $(1999)^{22}$ & & 8 & 3 & 8 & & 11 & 36 \\
\hline \multicolumn{8}{|l|}{ Stage IIA (T1-2N1) } \\
\hline Rosenthal $(1992)^{24}$ & & & & & $55^{\mathrm{d}}$ & & 30 \\
\hline \multicolumn{8}{|l|}{ Stage I \& IIA (T1-2N0-1) } \\
\hline Schildge $(1993)^{27}$ & & & & 30 & & & 28 \\
\hline Furuta $(1996)^{30}$ & & $25^{\mathrm{d}}$ & & & & & $25^{\mathrm{d}}$ \\
\hline \multirow{2}{*}{ Hayakawa $(1996)^{31}$} & Tumour $<5 \mathrm{~cm}$ & & & & $24^{a}$ & & 57 \\
\hline & Tumour $>5 \mathrm{~cm}$ & & & & $53^{a}$ & & 80 \\
\hline \multicolumn{8}{|l|}{ Stage I E IIB (T1-3NO) } \\
\hline \multirow[t]{4}{*}{ Dosoretz $(1992)^{36}$} & & 70 & & & & & 45 \\
\hline & $T 1$ & $30^{c}$ & & & & & $9^{c}$ \\
\hline & $T 2$ & $80^{c}$ & & & & & $40^{\circ}$ \\
\hline & T3 & $86^{c}$ & & & & & $40^{\circ}$ \\
\hline \multirow[t]{3}{*}{ Kupelian $(1996)^{39}$} & $T 1$ & 11 & & & & & \\
\hline & $T 2$ & 39 & & & & & \\
\hline & T3 & 58 & & & & & \\
\hline
\end{tabular}

Sites of failure according to whether noted to be the first site of relapse, an isolated site of relapse, or a site of relapse at any time during the course of the disease. Results of subgroup analyses are shown in italics.

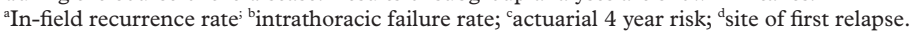


Mediastinal irradiation: isolated relapse in the hilum or mediastinum was uncommon $(0-3 \%)$ in those studies of stage I tumours in which the mediastinum had not been irradiated $^{1617192122}$; regional relapse occurred more commonly in association with local or distant failure $(6-15 \%)$. In studies where the mediastinum had been included in a proportion of cases $^{212239}$ survival was not improved by the use of mediastinal irradiation, although this comparison could well be biased by the use of mediastinal irradiation in higher risk patients.

Locoregional relapse was significantly less likely in one study if those with stage II tumours had received at least 40 Gy to the mediastinum, although the effect on survival was of borderline significance. ${ }^{31}$ However, in patients with stage I tumours, survival appeared to be better if the mediastinum had been treated, ${ }^{18}$ although the complete response rate was higher in this group.

Distant metastases: the incidence of distant metastases ranged from $16 \%$ to $40 \%$ (median $24 \%$ ) in studies reporting only stage I tumours and from $25 \%$ to over $50 \%$ in those which included stage IIA/B tumours (table 5). The highest incidence was reported in the study by Hayakawa $^{31}$ where $57 \%$ of those with tumours less than $5 \mathrm{~cm}$ in diameter and $80 \%$ of those with tumours over $5 \mathrm{~cm}$ in diameter developed distant metastases. Distant metastases were observed more frequently ( $52 \% v 12 \%)$ when a complete response had not been obtained. ${ }^{16}$ The rising incidence of metastases with advancing $T$ stage was no longer apparent if local control had not been achieved. ${ }^{36}$

Quality of life: although not defined as end points within this review, information was sought about symptom control and quality of life. No study reported on the impact of treatment on symptoms, quality of life, or performance status.

\section{Morbidity}

Spinal cord damage: one patient in the study by Zhang et $a l^{25}$ died from radiation myelopathy but no further details are given.

Lung damage: lung toxicity was reported in several studies with the highest incidence of pneumonitis or lung damage (not graded) being seven of 38 (18\%). ${ }^{11}$ In the two studies that used RTOG scales (also the two studies using hyperfractionation $)^{19}{ }^{21}$ there was grade 3 pneumonitis in $1-2 \%$ of patients and grade 3 late lung toxicity in $0-2 \%$ with one case of fatal pneumonitis. The use of doses in excess of 80 Gy (delivered in 2 Gy fractions) for central tumours resulted in four out of five patients developing bronchial stenosis. ${ }^{31}$ In the absence of prospective data recording, morbidity is likely to have been underestimated in a number of studies. Only one study reported pretreatment lung function tests $\left(\mathrm{FEV}_{1}\right){ }^{33}$ Posttreatment $\mathrm{FEV}_{1}$ was not reported.

\section{Discussion}

It is always preferable to base clinical decisions on the results of randomised trials. In the absence of these, one must look to results from observational studies although these represent a lower level of evidence. While evidence from non-randomised studies may be subject to several types of bias, there is evidence that patients entered into randomised trials may not reflect patients at large (fewer patients with adverse prognostic factors may be recruited into trials). On the other hand, non-randomised studies may appear to overestimate the effect of treatment because of poorer results in "historical" control groups. ${ }^{50}$ The result is that the magnitude of reported treatment effects tends to be fairly similar. ${ }^{51}$ This is borne out in this review where the 2 year survival rates in the two arms of the CHART trial (24-37\%) lie in the range reported for the non-randomised studies $(22-40 \%)$ of patients with stage I/IIA disease.

This review has attempted to categorise the patient populations in these non-randomised studies in order to draw conclusions about outcome and prognostic factors. Many of these retrospective reviews are relatively small so that, when outcomes for individual subgroups are examined, tests for statistical significance become inappropriate. Inevitably, there are many possibilities for bias affecting these conclusions but, hopefully, this systematic review will help to identify key questions to be addressed in future randomised trials.

For stage I/II NSCLC the effectiveness of radiotherapy relative to surgery remains uncertain. The only comparison of surgery and radiotherapy comes from a single randomised trial $^{5}$ performed 40 years ago when standards and techniques of staging and radiotherapy were very different. For patients considered "medically inoperable", a comparison with surgery would be inappropriate and unrealistic as almost $90 \%$ had been considered not sufficiently fit for surgery.

There have, unfortunately, been no randomised trials that have addressed the key question of whether patients with medically inoperable NSCLC should receive immediate radical radiotherapy or be watched and only receive palliative radiotherapy when they develop symptoms warranting treatment. A randomised trial of immediate versus delayed palliative radiotherapy did not show a difference in symptom control or survival but included only patients with locally advanced disease.$^{52}$ Indirect evidence supporting the use of radiotherapy (variation in survival according to radiotherapy technique and other subgroup analyses) has been sought.

\section{PATIENT SELECTION}

The proportion of patients declining surgery varied between studies. As these patients had been offered surgery, they probably had less comorbidity and better performance status than those considered unfit for surgery. As outcome is highly dependent on performance status, weight loss, and comorbidity, the size of this group of patients may have affected the outcome in individual series. The nonrandomised study with the best survival rates was the one with the highest proportion of patients declining surgery and with Karnofsky scores of $90-100^{19}$; because of this it is difficult to gauge whether the use of hyperfractionation 
in this study may have contributed to the results.

IMPACT OF CLINICAL $V$ PATHOLOGICAL STAGING Prior to the advent of CT imaging, $37 \%$ of patients were upstaged at surgery. ${ }^{53}$ In a more recent randomised study of lobectomy versus limited resection in patients with $\mathrm{T} 1 \mathrm{~N} 0$ NSCLC, ${ }^{54} 21 \%$ were found to have more advanced disease at surgery. This difference between clinical and pathological staging is recognised in the supporting evidence to the revised staging system. ${ }^{2}$ For example, 5 year survival was $57 \%$ for pathologically staged T2N0 (stage IB) tumours but only $38 \%$ for those clinically staged. Awareness of these survival differences suggests that the gap between 5 year survival rates for surgery and radiotherapy may not be as wide as at first appears.

IMPACT OF COMORBIDITY

The "gap" in effectiveness between surgery and radiotherapy may be further narrowed when factors which make surgery ill advised such as coexistent medical conditions or general frailty are taken into consideration. In this review deaths from causes other than cancer occurred in approximately one quarter of all patients treated. In contrast, in a retrospective study of 289 resected patients there were 79 non-cancer deaths $(27 \%),{ }^{55}$ and in the previously cited randomised trial of lobectomy versus limited resection 35 of the 247 patients $(14 \%)$ died from causes other than cancer. ${ }^{54}$ In this review poor respiratory function was the cause for inoperability in almost half of those judged not fit for surgery, and in a study of patients with chronic obstructive pulmonary disease (COPD) but without a diagnosis of cancer only $41 \%$ of those with an $\mathrm{FEV}_{1}$ less than $50 \%$ of predicted were alive at 5 years. ${ }^{56} \mathrm{It}$ is therefore difficult to assess the impact of comorbidity in different studies, especially as retrospective studies may underestimate noncancer deaths to a greater extent than prospective or randomised studies and as the proportion of non-cancer deaths is also related to duration of follow up.

\section{TREATMENT OUTCOME}

There is a difference of approximately $10-20 \%$ between overall and cancer specific survival at 5 years (table 3 ). Despite the poor overall survival of this group of patients, there are subgroups who may benefit to a greater degree and subgroups whose outcome is so poor that alternative approaches should be considered. The former group includes those with $\mathrm{T} 1$ tumours, of whom more than one third survive 5 years. The latter group includes those with significant weight loss or poor performance status for whom median survival times may be substantially less than 1 year.

Age alone did not appear to be a significant prognostic factor, although one might expect older patients to have a greater incidence of coexistent cardiorespiratory disease which, in turn, might mitigate against a better outcome.
Response rates have been reported as they may give some indication of treatment effectiveness. However, it is well recognised that assessment is more difficult for central than for peripheral tumours, where there is associated collapse or consolidation, and that assessment by plain radiography alone is less reliable than by CT imaging. The presence of adjacent pneumonitis or fibrosis frequently makes interpretation difficult. In this instance, local progression free survival would be a better measure of local control.

With respect to local failure, there is the need to distinguish between recurrence at the primary site, recurrences that are regional (that is, in hilar and mediastinal nodes), and recurrences elsewhere in the chest (which may be regarded as a category of systemic relapse). For locoregional failure it is important to know whether failure was within the radiation field, although this was not often stated. Reports tended to define local failure differently and table 4 may therefore have inadvertently included figures under the same category where there was a mixture of local and locoregional failure. Such data need to be collected prospectively, ideally within the context of a randomised trial.

\section{RADIATION DOSE}

The CHART trial clearly demonstrates the superiority of the accelerated and hyperfractionated CHART regime over conventionally fractionated radiotherapy to $60 \mathrm{~Gy} .{ }^{6}$ However, most of the patients in the stage I/IIA subgroup analysis had T2N0 disease. ${ }^{7}$ The relative absence of patients with T1N0 disease may reflect the reluctance of clinical oncologists to employ a technique using elective mediastinal irradiation for small tumours, especially if peripheral.

Although radiation dose appeared to influence outcome in several non-randomised studies, factors leading to choice of dose were rarely stated. It is possible that less fit patients or those with more advanced disease received a lower dose. In one study ${ }^{31}$ those showing signs of response received a higher dose. However, the presence of a dose-response relationship has been clearly demonstrated in a large randomised study of inoperable disease, ${ }^{57}$ so these observations may not be so lightly dismissed. A study of patients surviving at least 18 months following radiotherapy for stage I-III NSCLC found that there was a progressive fall in local failure rate with increasing dose such that, with a dose of $>59 \mathrm{~Gy}$, local failure occurred in only one of 20 patients. ${ }^{58}$ Similarly, a multivariate analysis of patients undergoing high dose palliative or radical radiotherapy showed the radiation dose to be a significant determinant of outcome. ${ }^{47}$

A recurring theme is that higher doses or more intensive regimes such as CHART may be associated with the best results. While CHART to $54 \mathrm{~Gy}$ is clearly superior to $60 \mathrm{~Gy}$ conventionally fractionated, one can only speculate on the possible benefits of dose escalation in both CHART and conventionally 
fractionated regimes without elective mediastinal irradiation.

MEDIASTINAL IRRADIATION

Traditionally, the whole mediastinum (extending from suprasternal notch to $5 \mathrm{~cm}$ inferior to the carina) is treated to a dose of 40-50 Gy in an attempt to eradicate subclinical disease. Most of the studies included in this review followed this practice. However, for peripherally located stage I tumours, isolated regional relapse was uncommon when the mediastinum was intentionally not included. Subclinical hilar or mediastinal node involvement in stage I/II NSCLC was found in one study to be less frequent in tumours not evident at bronchoscopy (that is, peripheral), ${ }^{59}$ and in a multivariate analysis tumour size was of only borderline significance. By not including the mediastinum, the mean lung dose will be lower and with it the risk of lung damage. ${ }^{60}$ As patients with stage I tumours referred for radiotherapy are commonly those with poor lung function, this would seem a reasonable approach.

For stage II tumours there may be fewer regional relapses if the mediastinum is treated, ${ }^{31}$ although an effect on survival was not apparent. The value of mediastinal irradiation for stage II tumours remains uncertain.

\section{DISTANT METASTASES}

This review found that only about one quarter of patients with stage I/II disease develop metastatic disease after radiotherapy, whereas this figure is in the region of $60-80 \%$ for those treated for stage III disease. The importance of this observation is that the potential for improving survival by improving local control is much greater in earlier stage disease.

In one study ${ }^{16}$ distant metastases occurred more frequently if a complete tumour response had not been obtained. Dosoretz et $a l^{36}$ reported that the incidence of distant metastases was related to tumour size only if there had been a complete response; without a complete response the relationship between tumour size and incidence of distant metastases was lost. While the biological properties of the tumour might predispose to both radioresistance and metastatic potential, these observations would also be consistent with persisting tumour continuing to act as a source of metastases.

\section{TREATMENT MORBIDITY}

In general, reporting of treatment side effects was inconsistent and mostly poor with little grading of adverse effects in retrospective studies. While there was prospective recording of morbidity in the CHART trial, data relating to patients with stage I/IIA disease have not been reported separately. There are therefore insufficient data to make a confident statement about treatment morbidity in this group.

USE OF CHEMOTHERAPY

Although the inclusion criteria for this review required that patients had been treated with radiotherapy alone rather than with a combination of radiotherapy and chemotherapy, in a meta-analysis which included patients with stages I-IIIB NSCLC the addition of adjuvant chemotherapy to radical radiotherapy improved 2 year survival from $15 \%$ to $19 \%$ and 5 year survival from $5 \%$ to $7 \% .{ }^{61}$ Synchronous chemoradiotherapy (the subject of a number of trials in patients with predominantly stage III disease) was not evaluated in this metaanalysis. Many medically inoperable patients in studies in this review were elderly and would not have been considered fit enough to receive chemotherapy. Whether synchronous chemoradiotherapy might offer any greater benefit without undue toxicity in this group has yet to be evaluated.

\section{Conclusions}

IMPLICATIONS FOR PRACTICE

As the majority of conclusions are based on the results of retrospective studies, the possibility of a significant degree of bias cannot be excluded.

- Radical radiotherapy offers the prospect of cure for a proportion of patients with medically inoperable NSCLC.

- Complete response rates are approximately $50 \%$ in tumours of $<4 \mathrm{~cm}$ in diameter but only $20 \%$ for tumours with a diameter of $>4 \mathrm{~cm} .{ }^{16} 18$

- Better outcome (overall survival) is seen in those with T1 tumours ${ }^{12} 1419202836$ and may be achieved with $\mathrm{CHART}^{7}$ or, where not available, conventionally fractionated doses of 60 Gy or more. ${ }^{12} 282937$

- Worse outcome is seen in those with: (a) performance status WHO/ECOG $\geqslant 2^{24} 31$ or Karnofsky score $\leqslant 80^{19}$; or

(b) weight loss of $\geqslant 5 \%$ body weight. ${ }^{19} 28$

- There is no evidence that age or histological subtype significantly affects outcome.

- The evidence does not support the use of mediastinal irradiation for peripheral stage I tumours. ${ }^{16} 1722$

- The benefits of mediastinal irradiation for centrally placed stage I tumours are uncertain.

- There is some evidence to support the use of mediastinal irradiation in stage II tumours. ${ }^{31}$

IMPLICATIONS FOR RESEARCH

- Randomised studies should help to determine the optimal dose and fractionation, in particular whether conventionally fractionated courses with dose escalation or CHART (or similar) with dose escalation offer benefits over CHART to 54 Gy.

- Studies are needed to determine the functional consequences of irradiating small fields in patients with poor lung function; this would help to establish evidence based criteria for eligibility for radical radiotherapy on the basis of lung function.

- Randomised studies are urgently needed to evaluate the practice of routine irradiation of clinically uninvolved sites within the mediastinum. In such a trial, patients with stage II or central stage I tumours (possibly with PET scanning to exclude mediastinal involvement) might be randomised to receive either radiotherapy to involved sites only or 
a two phase treatment (to the same total dose) with the first phase treating the whole mediastinum. Recording the mean lung dose and careful monitoring of lung function would be an essential part of such a study.

The authors are grateful to members of the Cochrane Cancer Network and the Cochrane Lung Cancer Review Group for their advice and support and to the BUPA Healthcare Develop-
ment Fund, Roy Castle Lung Cancer Foundation, Lilly Oncology, Zeneca, Ares Serono, Bristol Myers Squibb, Pierre Fabre Oncology, and Schering Plough for financial support. The support of Maidstone and Tunbridge Wells NHS Trust, Oxford Radcliffe NHS Trust, and Royal Marsden NHS Trust is also gratefully acknowledged. The authors are grateful to Dr F von Bueltzingsloewen for translation and to Dr R J O Davies for helpful comments.

A version of this review has been published in the Cochrane A version of this review has been published in the Cochrane
Library. Cochrane systematic reviews are regularly updated to include new research and in response to comments and criticism include new rese readers.

Conflicts of interest: None.

1 Royal College of Radiologists Clinical Oncology Information Network. Guidelines on the non-surgical management of lung cancer. Clin Oncol 1999;11:S23-8.

2 Mountain CF. Revisions in the International System for Staging Lung Cancer. Chest 1997;11:1710-7.

3 Vrdoljak E, Mise K, Sapunar D, et al. Survival analysis of untreated patients with non-small cell lung cancer. Chest 1994;106:1797-800.

4 World Health Organisation. Handbook for reporting results of cancer treatment. Geneva: WHO Offset Publications No 48, 1979.

5 Morrison R, Deeley TJ, Cleland WP. The treatment of carcinoma of the bronchus. A clinical trial to compare surgery and supervoltage radiotherapy. Lancet 1963;i:683-4.

6 Saunders M, Dische S, Barrett A, et al on behalf of the CHART Steering Committee. Continuous hyperfractionated accelerated radiotherapy (CHART) versus convenated accelerated radiotherapy (CHART) versus conventional radiotherapy in non-small cell lung cancer: mature data from the ran

7 Bentzen SM, Saunders MI, Dische S, et al. Updated data for CHART in NSCLC: further analyses. Radiother Oncol 2000;55:86-7.

8 Haffty BG, Goldberg NB, Gerstley J, et al. Results of radical radiation therapy in clinical stage I, technically operable non-small cell lung cancer. Int $\mathcal{F}$ Radiat Oncol Biol Phys 1988;15:69-73.

9 Noordijk EM, v d Poest Clement E, Hermans J, et al. Radiotherapy as an alternative to surgery in elderly patients with resectable lung cancer. Radiother Oncol 1988;13:83-9.

10 Sandler HM, Curran WJ, Turrisi AT. The influence of tumour size and pre-treatment staging on outcome following radiation therapy alone for stage I non-small cell lung cancer. Int $\mathcal{F}$ Radiat Oncol Biol Phys 1990 19:9-13.

11 Ono R, Egawa S, Suemasu K, et al. Radiotherapy in inoperable stage I lung cancer. fpn f Clin Oncol 1991;21:125-8.

12 Kaskowitz L, Graham MV, Emami B, et al. Radiation therapy alone for stage I non-small cell lung cancer. Int $\mathcal{f}$ therapy alone for stage I non-small cell

13 Slotman BJ, Njo KH, Karim ABMF. Curative radiotherapy for technically operable stage I nonsmall cell lung cancer. Int $\mathcal{F}$ Radiat Oncol Biol Phys 1994;29:33-7.

14 Gauden S, Ramsay J, Tripcony L. The curative treatment by radiotherapy alone of stage I non-small cell carcinoma of the lung. Chest 1995;108:1278-82.

15 Park JG, Won JH, Cha SI, et al. The prognosis following radiation therapy or surgical resection for stage I non-smal cell lung cancer. Tuberc Respir Dis 1995;42:731-6.

16 Krol ADG, Aussems P, Noordijk EM, et al. Local irradiation alone for peripheral stage I lung cancer: could we omit the elective regional nodal irradiation? Int 7 Radiat Oncol Biol Phys 1996;34:297-302.

17 Slotman BJ, Antonisse IE, Njo KH. Limited field irradiation in early stage $\left(\mathrm{T}_{1-2} \mathrm{~N}_{0}\right)$ non-small cell lung cancer. Radiother

18 Morita K, Fuwa N, Suzuki Y, et al. Radical radiotherapy for medically inoperable non-small cell lung cancer in clinical
stage I: a retrospective analysis of 149 patients. Radiother Stage I: a retrospective $1997 ; 42: 31-6$.

19 Jeremic B, Shibamoto Y, Acimovic L, et al. Hyperfractionated radiotherapy alone for clinical stage I non-small cell lung cancer. Int $\mathcal{F}$ Radiat Oncol Biol Phys 1997;38:521-5.

20 Nguyen-Tan PF, Chabot B, Beauchamp G, et al. Retrospective study of stage I non-small cell lung cancer treated by radiotherapy alone: does staging mediastinoscopy influence survival? Proc Am Soc Clin Oncol 1998;17:496a.

21 Sibley GS, Jamieson TA, Marks LB, et al. Radiotherapy alone for medically inoperable stage I non-small cell lung cancer: the Duke experience. Int $\mathcal{F}$ Radiat Oncol Biol Phys 1998;40:149-54.

22 Hayakawa K, Mitsuhashi N, Saito Y, et al. Limited field irradiation for medically inoperable patients with peripheral
stage I non-small cell lung cancer. Lung Cancer 1999;26: stage I non. $137-42$.

23 Salloum J. Radiation therapy a good alternative for unoperable NSCLC. f Miss State Med Assoc 1990;31:295-7.
24 Rosenthal SA, Curran WJ, Herbert SH, et al. Clinical stage II non-small cell lung cancer treated with radiation therapy alone. Cancer 1992;70:2410-7.

25 Zhang HX, Yin WB, Zhang LJ, et al. Curative radiotherapy of early operable non-small cell lung cancer. Radiother Oncol 1989;14:89-94.

26 Hayakawa K, Mitsuhashi N, Nakajima N, et al. Radiation therapy for stage I-III epidermoid carcinoma of the lung. Lung Cancer 1992;8:213-24.

27 Schildge J, Hinkelbein W, Freund U, et al. Die kurative strahlentherapie nichtklein-zelliger bronchialkarzinome der stadien I und II. Atemw-Lungenkrkh fahrgang 1993;4:145-

28 Graham PH, Gebski VJ, Langlands AO. Radical radiotherapy for early nonsmall cell lung cancer. Int $\mathcal{F}$ Radiat Oncol Biol Phys 1995;31:261-6.

29 Koukourakis M, Skarlatos J, Kosma L, et al. Radiotherapy alone for non-small cell lung cancer. Acta Oncol 1995;34: 525-30

30 Furuta M, Hayakawa K, Katano S, et al. Radiation therapy for stage I-II non-small cell lung cancer in patients aged 75 years and over. Fpn f Clin Oncol 1996;26:95-8.

31 Hayakawa K, Mitsuhashi N, Saito Y, et al. Definitive radiation therapy for medically inoperable patients with stage I and II non-small cell lung cancer. Radiat Oncol Invest 1996; 4:165-70.

32 Hayakawa K, Mitsuhashi N, Furuta M, et al. High-dose radiation therapy for inoperable non-small cell lung cancer without mediastinal involvement. Strahlenther Onkol 1996; 172:489-95.

33 King SC, Acker JC, Kussin PS, et al. High-dose, hyperfractionated, accelerated radiotherapy using a concurrent boost or the treatment of nonsmall cell lung cancer: unusual toxicity and promising early results. Int $\mathcal{F}$ Radiat Oncol Biol Phys 1996;36:593-9.

34 Anderson WJA, McAleer JJA, Stranex S, et al. Radical radiotherapy for inoperable non-small cell lung cancer: what factors predict prognosis? Clin Oncol 2000;12:48-52.

35 Talton BM, Constable WC, Kersh CR. Curative radiotherapy in non-small cell carcinoma of the lung. Int $\mathcal{F}$ Radiat Oncol Biol Phys 1990;19:15-21.

36 Dosoretz DE, Katin MJ, Blitzer PH, et al. Radiation therapy in the management of medically inoperable carcinoma of the lung: results and implications for future treatment strategies. Int $\mathcal{F}$ Radiat Oncol Biol Phys 1992;24:3-9.

37 Dosoretz DE, Galmarini D, Rubenstein JH, et al. Local control of medically inoperable lung cancer: an analysis of its importance in outcome and factors determining the probability of tumour eradication. Int $\mathcal{F}$ Radiat Oncol Biol Phys 1993;27:507-16.

38 Dosoretz DE, Katin MJ, Blitzer PH, et al. Medically inoperable lung carcinoma: the role of radiation therapy. Semin Radiat Oncol 1996;6:98-104.

39 Kupelian PA, Komaki R, Allen P. Prognostic factors in the treatment of node-negative nonsmall cell lung carcinoma with radiotherapy alone. Int $\mathcal{f}$ Radiat Oncol Biol Phys 1996; 36:607-13.

40 Hilton G. Present position relating to cancer of the lung Results with radiotherapy alone. Thorax 1960;15:17-8.

41 Smart J. Can lung cancer be cured by irradiation alone? ҰAMA 1966;195:1034-6.

42 Cooper JD, Pearson FG, Todd TRJ, et al. Radiotherapy alone for operable carcinoma of the lung. Chest 1985;87: 289-92.

43 Burt PA, Hancock BM, Stout R. Radical radiotherapy for carcinoma of the bronchus: an equal alternative to radical urgery? Clin Oncol 1989;1:86-90.

44 Newaishy GA, Kerr GR. Radical radiotherapy for bronchogenic carcinoma: five year survival rates. Clin Oncol 1989;1: $80-5$.

45 Reinfuss M, Skolyszewski J, Korzeniowski S, et al. Results of radiotherapy with curative intent in non-small cell lung cancer. Strahlenther Onkol 1992;168:573-8.

46 Wigren T, Kellokumpu-Lehtinen P, Ojala A. Radical radiotherapy of inoperable non-small cell lung cancer. Irradiation techniques and tumour characteristics in relation to local control and survival. Acta Oncol 1992;31:555-61.

47 Schaafsma J, Coy P. The effect of radiotherapy on the survival of non-small cell lung cancer patients. Int $\mathcal{F}$ Radiat Oncol Biol Phys 1998;41:291-8.

48 Willers $\mathrm{H}$, Wurschmidt $\mathrm{F}$, Bunemann $\mathrm{H}$, et al. High-dose radiation therapy alone for inoperable non-small cell lung cancer. Experience with prolonged overall treatment times. Acta Oncol 1998;37:101-5.

49 Koutaissoff S, Wellmann D, Coucke P, et al. Hyperfractionated accelerated radiotherapy (HART) for inoperable, nonmetastatic non-small cell lung carcinoma of the lung (NSCLC): results of a phase II study for patients ineligible for combination radiochemotherapy. Int f Radiat Oncol Biol Phys 1999;45:1151-6. 50 Sacks H, Chalmers TC, Smith H. Randomized versus
historical controls for clinical trials. Am $\mathcal{f}$ Med 1982;72: 233-40

51 Britton A, McKee M, Black N, et al. Choosing between randomised and non-randomised studies: a systematic review. Health Technology Assessment 1998;2:1-124.

52 Falk SJ, White RJ, Hopwood P, et al. Immediate versus delayed thoracic radiotherapy in patients with unresectable, locally advanced non-small cell lung cancer and minimal symptoms: results of an MRC/BTS randomised trial. Thorax 1999;54(Suppl 3):A14.

53 Martini N, Beattie EJ. Results of surgical treatment in stage I lung cancer. F Thorac Cardiovasc Surg 1977;74:499-505. 
54 Ginsberg RJ, Rubinstein LV for the Lung Cancer Study Group. Randomized trial of lobectomy versus limited resection for T1N0 no

55 Harpole DH, Herndon JE, Young WG, et al. Stage I nonsmall cell lung cancer. A multivariate analysis of treatment methods and patterns of recurrence. Cancer 1995;76: $787-96$.

56 Sahn SA, Nett LM, Petty TL. Ten year follow-up of a comprehensive rehabilitation program for severe COPD. Chest 1980;77(Suppl):311-4

57 Perez CA, Pajak TF, Rubin P, et al. Long-term observations of the patterns of failure in patients with unresectable nonoat cell carcinoma of the lung treated with definitive radiotherapy. Cancer 1987;59:1874-81.
58 Sherman DM, Weischelbaum R, Hellman S. The characteristics of long-term survivors of lung cancer treated with istics of long-term survivors of lun

59 Sawyer TE, Bonner JA, Gould PM, et al. Predictors of subclinical nodal involvement in clinical stages I and II non-small cell lung cancer. Int $\mathcal{f}$ Radiat Oncol Biol Phys 1999;43:965-70.

60 Kwa SLS, Lebesque JV, Theuws JCM, et al. Radiation pneumonitis as a function of mean lung dose: an analysis of pooled data of 540 patients. Int $\mathcal{F}$ Radiat Oncol Biol Phys 1998;42:1-9.

61 Non-Small Cell Lung Cancer Collaborative Group. Chemotherapy in non-small cell lung cancer: a metaanalysis using updated data on individual patients from 52 randomised clinical trials. BMf 1995;311:899-909.

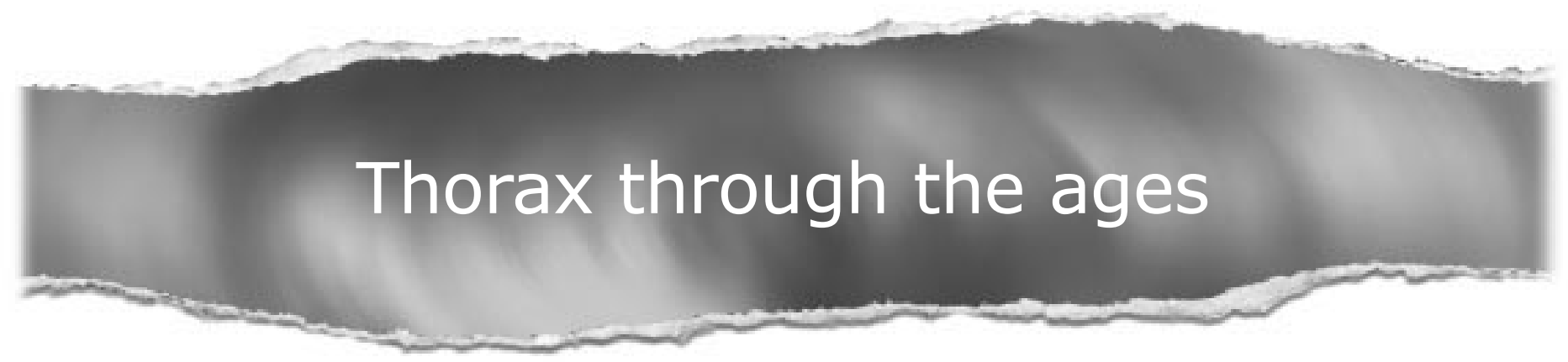

\section{Browse the Archive}

Thorax online has an archive of content dating back to 1966.

Full text from 1997; abstracts from 1975; table of contents from 1966

www.thoraxjnl.com 